\title{
Convalescent plasma therapy and mortality in COVID-19 patients admitted to the ICU: a prospective observational study
}

Stefan Hatzl ${ }^{1,2}$ (D), Florian Posch ${ }^{3}$, Nazanin Sareban ${ }^{4}$, Martin Stradner $^{5}$, Konrad Rosskopf ${ }^{4}$, Alexander C. Reisinger $^{1}$, Philipp Eller ${ }^{1}$, Michael Schörghuber ${ }^{6}$, Wolfgang Toller ${ }^{6}$, Zdenka Sloup $^{7}$, Florian Prüller ${ }^{7}$, Katharina Gütl $^{8}$, Stefan Pilz ${ }^{9}$, Alexander R. Rosenkranz ${ }^{10}$, Hildegard T. Greinix ${ }^{2}$, Robert Krause ${ }^{11^{*}}$, Peter Schlenke ${ }^{4}$ and Gernot Schilcher ${ }^{1}$

\begin{abstract}
Background: This study aimed to quantify the potential survival benefit of convalescent plasma therapy (CVP) in critically ill patients with acute respiratory failure related to coronavirus disease-2019 (COVID-19).

Methods: This is a single-center prospective observational cohort study in COVID-19 patients with acute respiratory failure. Immediately after intensive care unit (ICU) admission patients were allocated to CVP treatment following pre-specified criteria to rapidly identify those patients potentially susceptible for this treatment. A propensity score adjustment [inverse probability of treatment weighted (IPTW) analysis] was implemented to account rigorously for imbalances in prognostic variables between the treatment groups.
\end{abstract}

Results: We included 120 patients of whom 48 received CVP. Thirty percent were female with a median age of 66 years [25th-75th percentile 54-75]. Eighty-eight percent of patients presented with severe acute respiratory failure as displayed by a median $\mathrm{paO}_{2} / \mathrm{FiO}_{2}$ ratio (Horowitz Index) of 92 [77-150]. All patients required any kind of ventilatory support with more than half of them (52\%) receiving invasive ventilation. Thirty-day ICU overall survival (OS) was 69\% in the CVP group and 54\% in the non-CVP group (log-rank $p=0.049)$, respectively. After weighing the time-to-event data for the IPTW, the favorable association between CVP and OS became even stronger (log-rank $p=0.035$ ). Moreover, an exploratory analysis showed an overall survival benefit of CVP therapy for patients with non-invasive ventilation (Hazard ratio 0.12 95\% Cl 0.03-0.57, $p=0.007$ )

Conclusion: Administration of CVP in patients with acute respiratory failure related to COVID-19 is associated with improved ICU survival rates.

Keywords: COVID-19, ICU, Intensive care, Convalescent plasma, Respiratory failure, Acute respiratory distress syndrome

\footnotetext{
*Correspondence: robert.krause@medunigraz.at

${ }^{11}$ Section of Infectious Diseases and Tropical Medicine, Department

of Internal Medicine, Medical University of Graz, Graz, Austria

Full list of author information is available at the end of the article
}

\begin{abstract}
Background
In late fall of 2019 severe acute respiratory syndrome coronavirus 2 (SARS-CoV-2) emerged in Wuhan, China and has spread worldwide since then, infecting and killing millions of people. Coronavirus disease 2019 (COVID19), is caused by SARS-CoV-2 and represents a major challenge for global health-care systems and especially intensive care units (ICU) [1]. COVID-19 mainly affects
\end{abstract}


the respiratory system with some patients rapidly progressing to acute respiratory distress syndrome (ARDS) [2]. Since the outbreak, various treatment strategies have been investigated with only a minority of them providing convincing benefits [3-7].

Use of convalescent plasma (CVP) as "passive immunization" has a history going back to the 1890s and was the only method of treating some infectious diseases before the development of antimicrobial therapies [8]. The rationale behind this approach is to jump start the immune system with a passive antibody therapy in the combat against SARS-CoV-2 [9, 10]. Large observational studies demonstrated an adequate safety profile for SARS-CoV-2 CVP [11].

Despite the increasing number of clinical trials investigating CVP against SARS-CoV-2 infection, the effect of CVP on COVID-19 patient outcomes remain elusive so far including the timing and selection of patients for optimal treatment efficacy [12-17]. In this context, there is accumulating evidence that early administration of CVP and certain patient characteristics may be main determinants for treatment success of CVP in COVID-19 patients $[13,15,17,18]$. Nevertheless, significant knowledge gaps remain on this issue, in particular, in terms of which specific patient groups are those with particular treatment success in response to CVP therapy for COVID-19.

Within this prospective case-control study, we aimed to evaluate the effects of CVP therapy on overall survival (OS) in critically ill patients primarily admitted to the ICU due to COVID-19 associated acute respiratory failure. Immediately after ICU admission, CVP treatment was administered in patients who were selected for this therapy based on pre-specified criteria that aimed to identify patients who did not yield anti-SARS-CoV-2 antibody conversion. Therefore, our main rationale was that progression of lung injury and death from COVID19 might originate from the SARS-CoV-2 viral burden which cannot be cleared due to an insufficient host immune response characterized by the absence of antiSARS-CoV-2 antibodies and that supplementation of those antibodies by CVP should lead to clinical benefit.

\section{Methods}

\section{Study cohort}

For this prospective exposed-non-exposed cohort study (where exposed are the patients who received CVP and non-exposed those who did not), we considered all 757 consecutive SARS-CoV-2 polymerase chain reaction (SARS-CoV-2 PCR) positive patients who were referred for the treatment of COVID-19 at the Department of Internal Medicine, Medical University of Graz, Austria, between March 13th, 2020 and Jan 5th, 2021. All patient data was prospectively and uniformly collected according to a guidance which was locally released in March 2020 as a standard operating procedure (SOP) at our department. Laboratory and radiology data were extracted from our in-house electronic healthcare database system as described previously [19]. Final analysis (Fig. 1a) was performed after exclusion of patients who had not been admitted to the ICU $(n=586)$ or had received prior CVP therapy $(n=4)$, patients with non-pulmonary reason for ICU admission $(n=30)$, patients with insufficient followup data $(n=15)$ and patients with pulmonary P. jirovecii (PJP) co-infection $(n=2)$. In- and exclusion criteria are tabulated in Fig. 1b. The research project was approved by the local institutional review board (EK-Nr.: 32-475 ex 19/20).

\section{Indication and rational for CVP administration}

Indication and administration of CVP has been informed by a standard operating procedure (SOP) which was published in Mach 2020 as local guidance for the Department of Internal Medicine, Medical University of Graz, Austria. Allocation of CVP therapy was made by an attending ICU or ID (infectious disease) physician. CVP therapy was restricted to COVID-19 patients admitted to ICU due to acute respiratory failure with acquired (after chemotherapy, under immunosuppression) or inborn immune deficiency (for detailed information about the immunosuppression see Additional file 1) or patients with negative SARS-CoV-2 antibody status at ICU admission.

\section{Evaluation of anti-SARS-CoV-2 antibodies in patients}

To evaluate the serological status of patients admitted to ICU, total antibodies (including IgG and IgM) against SARS-CoV-2 nucleocapsid were measured using the Roche Elecsys ${ }^{\circledR}$ Anti-SARS-CoV-2 immunoassay (Roche Diagnostics, Rotkreuz, Switzerland) on a Cobas e801 analyzer platform (specificity $>99 \%$ ) as described previously [20]. A cut-off index $>1$ was regarded as positive.

\section{CVP collection and donor evaluation}

COVID-19 convalescent apheresis plasma was collected at the Department of Blood Serology and Transfusion Medicine, Medical University Graz, Austria, between April 10th and November 11th 2020. Only clinical fully recovered COVID-19 patients donated plasma according to the recommendations of the European Commission and standard operating procedures of our department after written informed consent. To ensure therapeutic potential of CVP a minimum of $30 \mathrm{AU} / \mathrm{ml}$ neutralizing anti-SARS-CoV-2 S1/S2 IgG antibodies (DiaSorin ${ }^{\circledR}$, Vienna, Austria) were determined as a release criterion of CVP. CVP was produced according to good 


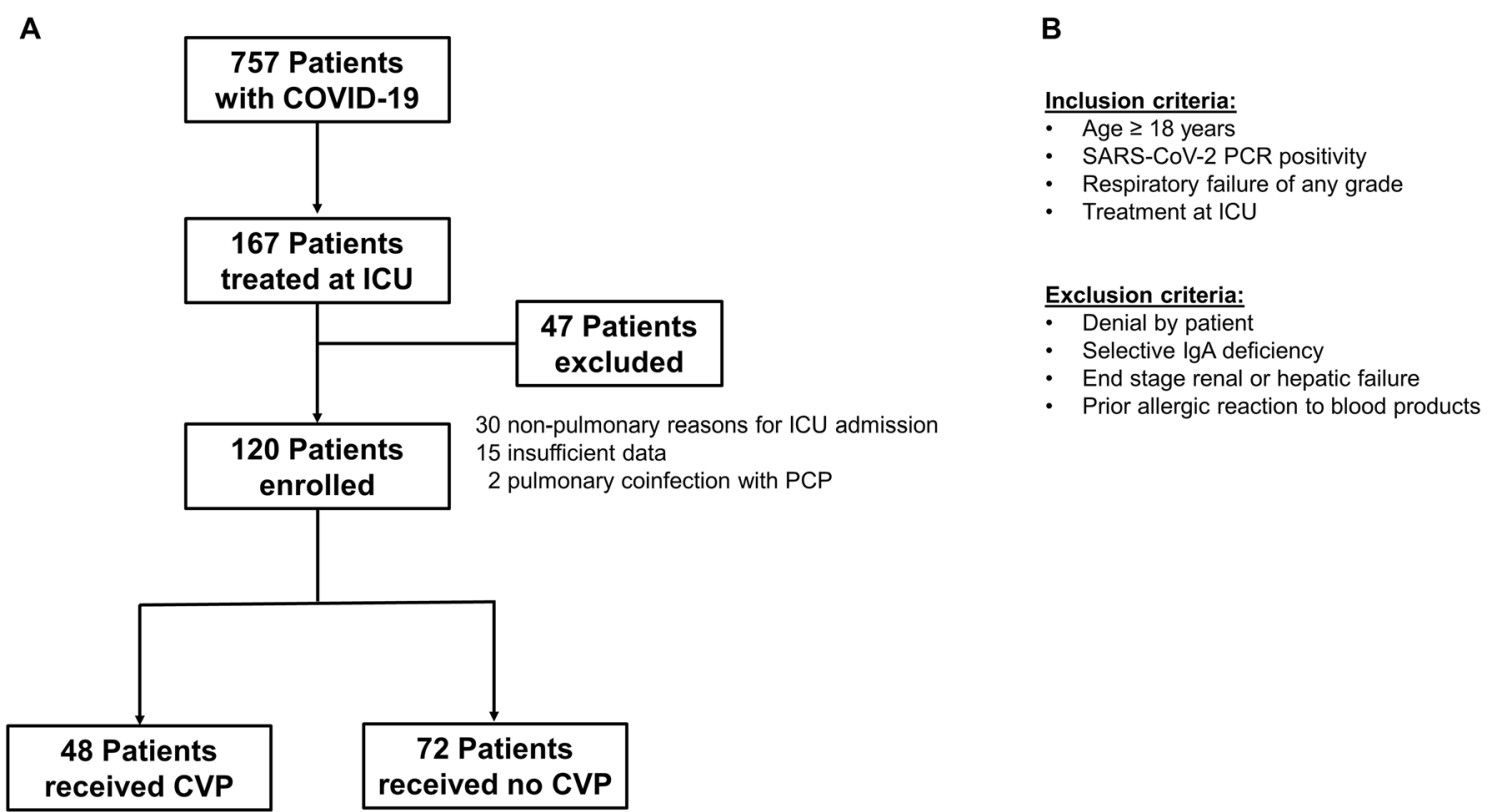

Fig. 1 Full trial protocol and flow diagram. a Recruitment tree: 757 patients were initially considered for the study. 167 were treated at intensive care units (ICU). 47 patients were excluded - thereof 30 patients were excluded because of other than pulmonary indications for ICU admission. SARS-CoV-2 positivity was an incidental finding without any radiologic evidence of pneumonia. 15 were treated due to non-ST or ST-elevation myocardial infarction (NSTEMI/STEMI); 11 received post-operative intensive care treatment-3 patients were incidentally tested positive for SARS-CoV-2 due to ischemic or hemorrhagic stroke; 1 patient was incidentally tested positive for SARS-CoV-2 after cerebral bleeding within the work-up for organ donation, 15 patients were excluded due to insufficient follow-up data and 2 patients were excluded, because they had pulmonary co-infection with pneumocystis jirovecii (PJP). b Inclusion and exclusion criteria of the study. SARS-CoV-2 severe acute respiratory syndrome corona virus 2; PCR: polymerase chain reaction; ICU: intensive care unit; IgA: immunoglobulin A

manufacturing practice (GMP) guidelines and additional pathogen inactivation was performed using INTERCEPT Blood System (Cerus E.V., Amersfoort, The Netherlands) which is described in more detail in Additional file 2.

\section{Statistical analysis}

All statistical analyses were performed using Stata (Windows version 15.1; Stata Corp., Houston, TX, USA). The distribution of baseline variables between the CVP and non-CVP group was evaluated using rank-sum tests, $X^{2}$ tests, and Fisher's exact tests, as appropriate. The magnitude of these differences was quantified with standardized mean differences (SMDs), and SMDs $\geq 0.30$ were considered indicative of a potentially relevant magnitude of difference. We obtained the propensity score $e$ from a multivariable logistic regression model with treatment group as the outcome variable. For this model, we prespecified a fixed number of 10 predictor variables, in order not to have less than 5 events per predictor variable. In detail, the model was developed by initially including all variables with a $p$ for difference between the two groups of $\leq 0.10$ and $S M D \geq 0.30$. The following variables were excluded from the model development process: (1) mSOFA (modified sequential organ failure assessment) score and acute respiratory failure grading (inspired by acute ARDS Berlin 2012 classification) (due to strong collinearity with the Horowitz index); (2) white blood count (due to strong collinearity with the neutrophil count); and (3) the SARS-CoV-2 antibody status variable (due to the so-called perfect prediction problem), and the immunosuppression variable (due to the introduction of model instability by this variable). This model was then reduced with simple backward elimination to yield the pre-specified 10 predictor variable model. The propensity score $e$ was then transformed into an inverse-probability-of-treatment-weight (IPTW) according to the average treatment effect principle, i.e., $=\frac{\mathrm{CVP}}{e}+\frac{1-\mathrm{CVP}}{1-e}$, where CVP denotes the treatment assignment $(0=$ nonCVP group, $1=$ CVP group) [21]. Following best practice recommendations for balance diagnostics, we then re-estimated SMDs and $p$ values for difference between the two treatment groups with the IPTW-weighted data [22, 23]. The primary outcome of the study, 3-month overall survival, was defined as the time interval from ICU admission to death-from-any-cause or the censoring date when being still alive 3 months after ICU admission. The 30-day ICU survival was defined as co-primary outcome to get further insights in the early ICU mortality 
of COVID-19 patients. Survival time was inflated by 1 day in patients who died at the day of ICU admission $(n=5)$. OS was computed with Kaplan-Meier estimators, and compared between the two treatment groups using log-rank tests. Uni- and multivariable modelling of mortality hazards was performed with Cox regression models. No evidence for a violation of the proportional hazards assumption according to treatment assignment was observed. These time-to-event analyses were then re-performed with IPTW weighting to control for selection bias. Sensitivity analyses included a trimmed IPTW (i.e., excluding patients with an IPTW $\leq$ the 1 st and $\geq$ the 99th percentile of its distribution), and multivariable adjustment for variables that had high SMDs after IPTW weighting. In an exploratory hypothesis generating analysis we fitted interactions between treatment assignment and pre-specified subgroups (age $\leq />65$ years, number of comorbidities $\leq />3$, mSOFA $\leq />5$ points, $\mathrm{paO}_{2} /$ $\mathrm{FiO}_{2} \leq />100$, and Ferritin $\leq />1500 \mathrm{mg} / \mathrm{dl}$ ) to gauge whether the "effect" of CVP on OS may be modified by these clinical co-variables. The full dataset and the main analysis code are available on request by the first author.

\section{Results}

\section{ICU cohort and CVP administration}

One hundred twenty patients were admitted to the ICU due to COVID-19 associated acute respiratory failure, with 48 (40\%) patients receiving CVP immediately after ICU admission according to our local standard (Table 1, Fig. 1). At the time of ICU admission, the median age of the cohort was 66 years [25th-75th percentile: 54-75], and $36(30 \%)$ patients were female. The study population had a median of three comorbidities [1-4] and a median time from COVID-19 symptom onset to ICU admission of 4.0 days [1-6]. All patients included in this study showed an oxygen saturation of less than $88 \%$ while breathing ambient air prior to ICU admission. Most patients presented with moderate to severe respiratory failure as displayed by a median $\mathrm{paO}_{2} / \mathrm{FiO}_{2}$ ratio (Horowitz Index) of 92 [77-150]. The median antiSARS-CoV-2 antibody concentration in CVP was 79.2 $\mathrm{AU} / \mathrm{ml}$ [46.6-99.7], with a median dose of $600 \mathrm{ml}$ CVP [600-600] administered in two applications (400 $\mathrm{ml}$ day $1,200 \mathrm{ml}$ day 2) or three subsequent daily doses of $200 \mathrm{ml}$ depending on fluid tolerance of the patients. During a median follow-up of 1.9 months, we observed 52 deaths, corresponding to 1-, 2-, and 3-month OS estimates of $60 \%$ (95\% CI 51-68), 54\% (45-63), and 54\% (45-63), respectively, in the complete cohort comprising 120 patients. (Additional file 3). Importantly, no unexpected or serious adverse events related to CVP administration were observed during the study period thereby encouraging the previously published outstanding safety profile of this therapy additionally regarding usage in ICUs.

\section{Overall survival according to CVP administration-crude analysis}

In a first step we evaluated our CVP allocation strategy by anti-SARS-CoV-2 anybody status at ICU admission and/or history of inborn or acquired immunosuppression. Therefore, we performed an unadjusted "crude" analysis of our data.

In crude analysis, patients given CVP experienced a more favorable 3-month OS than patients who did not receive CVP. In detail, after 1.3 months $50 \%$ of patients deceased in the non-CVP group, whereas patients allocated to CVP treatment did not reach this threshold (log-rank $p=0.049$, Fig. 2a). To get further insights into the early ICU mortality, we calculated the 30-day OS which estimates $69 \%$ in the CVP group, and 54\% in the non-CVP group. In univariable Cox regression, this corresponded to a Hazard Ratio (HR) of 0.56 (95\% CI $0.31-$ $1.01, p=0.054)$ by CVP therapy.

\section{Development of a propensity score}

On average, patients who received CVP therapy had baseline covariates consistent with more severe critical illness (Table 1, Additional file 4). Specifically, as indicated by standardized mean differences (SMD), patients in the CVP therapy group had a higher number of comorbidities, a higher modified sequential organ failure assessment (mSOFA) score, a lower $\mathrm{paO}_{2} /$ $\mathrm{FiO}_{2}$ ratio, more severe acute respiratory failure grade (inspired by ARDS Berlin 2012-classification), and higher levels of adverse predicting laboratory parameters such as lactate, interleukin-6, and C-reactive protein. Otherwise, patients in the CVP group were significantly younger and less likely to be treated in the "first wave" of COVID-19 (March-May 2020), and (consistent with the local guidance for CVP administration) had a higher prevalence of immunosuppression and negativity for SARS-CoV-2 antibodies (Table 1). These imbalances are consistent with the non-random assignment to CVP by treating physicians, likely underestimating potential beneficial effects of CVP therapy (i.e., a "conservative" bias). To control for this potential bias, we predicted a propensity score based on a 10-variable multivariable logistic regression model (Table 2). The propensity score covered the whole probability range (Additional file 5-Panel A) and was then transformed into the IPTW (Additional file 5-Panel B). Re-weighting of the data strongly reduced many but not all differences in baseline covariates between the two treatment groups (Table 1, Additional file 6), which 
Table 1 Baseline characteristics of the study population—distribution at ICU admission

\begin{tabular}{|c|c|c|c|c|c|}
\hline Variable & Overall $(n=120)$ & $\operatorname{CVP}(n=48)$ & Non-CVP $(n=72)$ & $p$ & $p_{\text {IPTW }}$ \\
\hline \multicolumn{6}{|l|}{ Demographic variables } \\
\hline Age (years) & $66[54-75]$ & $61[53-72]$ & $69[55-76]$ & 0.044 & 0.724 \\
\hline Female Gender & $36(30 \%)$ & $12(25 \%)$ & $24(33 \%)$ & 0.417 & 0.277 \\
\hline BMI $\left(\mathrm{kg} / \mathrm{m}^{2}\right)$ & $27.7[24.5-32.5]$ & $29.8[25.0-34.4]$ & $27.1[23.9-31.3]$ & 0.058 & 0.558 \\
\hline "First COVID-19 wave" & $28(23 \%)$ & $3(6 \%)$ & $25(35 \%)$ & $<0.001$ & 0.194 \\
\hline \multicolumn{6}{|l|}{ Coexisting conditions } \\
\hline Number of coexisting conditions & $3[1-4]$ & $3[2-5]$ & $2[1-4]$ & 0.019 & 0.905 \\
\hline Hypertension & $94(78 \%)$ & $43(90 \%)$ & $51(70 \%)$ & 0.022 & 0.838 \\
\hline Diabetes & $46(38 \%)$ & $23(48 \%)$ & $23(33 \%)$ & 0.088 & 0.825 \\
\hline Atrial fibrillation & $24(20 \%)$ & $15(31 \%)$ & $9(13 \%)$ & 0.019 & 0.163 \\
\hline Coronary heart disease & $31(26 \%)$ & $14(29 \%)$ & $17(24 \%)$ & 0.520 & 0.457 \\
\hline Congestive heart failure & $30(25 \%)$ & $17(24 \%)$ & $13(27 \%)$ & 0.672 & 0.738 \\
\hline Peripheral arterial disease & $23(19 \%)$ & $15(21 \%)$ & $8(17 \%)$ & 0.641 & 0.195 \\
\hline Thromboembolic disease & $18(15 \%)$ & $10(21 \%)$ & $8(11 \%)$ & 0.132 & 0.680 \\
\hline Chronic renal failure & $35(24 \%)$ & $18(38 \%)$ & $17(24 \%)$ & 0.107 & 0.994 \\
\hline Dialysis & $9(8 \%)$ & $3(6 \%)$ & $6(8 \%)$ & 0.740 & 0.104 \\
\hline COPD & $16(13 \%)$ & $10(14 \%)$ & $6(13 \%)$ & 1.000 & 0.742 \\
\hline Asthma & $13(10 \%)$ & $8(17 \%)$ & $5(7 \%)$ & 0.130 & 0.498 \\
\hline Prior cancer in complete remission & $13(10 \%)$ & $8(17 \%)$ & $5(7 \%)$ & 0.507 & 0.226 \\
\hline Active malignancy & $9(8 \%)$ & $5(10 \%)$ & $4(6 \%)$ & 0.105 & 0.228 \\
\hline Dementia & $4(3 \%)$ & $2(4 \%)$ & $2(3 \%)$ & 1.000 & 0.947 \\
\hline Prior organ transplantation & $5(4 \%)$ & $5(10 \%)$ & $0(0 \%)$ & 0.009 & N/E \\
\hline Immunosuppression $^{\dagger}$ & $18(15 \%)$ & $15(30 \%)$ & $3(4 \%)$ & $<0.001$ & 0.009 \\
\hline \multicolumn{6}{|l|}{ ICU risk stratification } \\
\hline mSOFA (points) & $5[4-7]$ & $6[4-8]$ & $4[3-5]$ & $<0.001$ & 0.204 \\
\hline $\mathrm{paO}_{2} / \mathrm{FiO}_{2}$ & $92[77-150]$ & 81 [71-96] & $113[83-186]$ & $<0.001$ & 0.198 \\
\hline PEEP $(\mathrm{mmHg})$-maximum & $11[9-12]$ & $11[9-13]$ & $10[9-12]$ & 0.157 & 0.623 \\
\hline $\begin{array}{l}\text { Acute respiratory failure grade (inspired by ARDS Berlin } \\
\text { 2012-classification) }\end{array}$ & / & / & / & $<0.001$ & 0.140 \\
\hline Severe & $76(63 \%)$ & $42(88 \%)$ & $34(47 \%)$ & / & / \\
\hline Moderate & $30(25 \%)$ & $6(13 \%)$ & $24(33 \%)$ & / & / \\
\hline Mild & $14(12 \%)$ & $0(0 \%)$ & $14(19 \%)$ & / & / \\
\hline Ventilation (maximum invasivity) & / & / & / & 0.400 & 0.364 \\
\hline Intubated & $55(46 \%)$ & $25(52 \%)$ & $30(42 \%)$ & / & / \\
\hline vvECMO & $7(6 \%)$ & $3(6 \%)$ & $4(6 \%)$ & / & / \\
\hline NIV & 47 (39\%) & $18(38 \%)$ & $29(40 \%)$ & / & / \\
\hline HFNC & $11(9 \%)$ & $2(4 \%)$ & $9(13 \%)$ & / & / \\
\hline Any invasive ventilation & $62(52 \%)$ & $28(58 \%)$ & $34(47 \%)$ & 0.233 & 0.130 \\
\hline \multicolumn{6}{|l|}{ CVP characteristics } \\
\hline Length of symptoms before first transfusion (days) & / & $4[1-10]$ & / & / & \\
\hline Antibody concentration (AU/ml) & / & $79.2[46.6-99.7]$ & / & / & \\
\hline Administered dose of CVP (ml) & / & $600[600-600]$ & / & / & \\
\hline \multicolumn{6}{|l|}{ Laboratory values } \\
\hline Lactate (mmol/l) & $2.7[1.6-3.8]$ & $2.9[2.3-4.1]$ & $1.9[1.2-3.1]$ & 0.003 & 0.646 \\
\hline $\mathrm{IL}-6(\mathrm{pg} / \mathrm{ml})^{*}$ & $125[65-245]$ & $171[85-334]$ & 104 [40-204] & 0.017 & 0.680 \\
\hline $\mathrm{CRP}(\mathrm{mg} / \mathrm{l})$ & 110 [63-187] & 145 [104-200] & $96[42-169]$ & $<0.001$ & 0.157 \\
\hline Ferritin $(\mathrm{ng} / \mathrm{ml})^{*}$ & 1240 [523-2090] & 1940 [799-2829] & 910 [456-1479] & $<0.001$ & 0.028 \\
\hline $\mathrm{hs}-\mathrm{TnT}(\mathrm{pg} / \mathrm{ml})^{*}$ & $18[9-31]$ & $18[8-26]$ & $19[11-54]$ & 0.200 & 0.263 \\
\hline D-Dimer $(m g / l)^{*}$ & $2.3[1.1-4.8]$ & $2.5[1.4-5.4]$ & $2.0[0.9-4.7]$ & 0.172 & 0.484 \\
\hline SARS-CoV-2-Antibody positivity at ICU admission & $49(49 \%)$ & $3(7 \%)$ & $46(85 \%)$ & $<0.001$ & 0.001 \\
\hline
\end{tabular}


Table 1 (continued)

\begin{tabular}{|c|c|c|c|c|c|}
\hline Variable & Overall $(n=120)$ & $\operatorname{CVP}(n=48)$ & Non-CVP $(n=72)$ & $p$ & $\overline{p_{\text {IPTW }}}$ \\
\hline \multicolumn{6}{|l|}{ Blood counts } \\
\hline Leukocytes [G/l] & $8.3[5.1-11.4]$ & $6.0[4-1-10.1]$ & 8.7 [6.3-12.3] & 0.007 & 0.020 \\
\hline Neutrophiles [G/l] & $6.5[2.4-9.1]$ & $4.6[3.0-7.6]$ & $7.2[4.0-9.4]$ & 0.005 & 0.042 \\
\hline Lymphocytes [G/l] & $0.7[0.5-1.2]$ & $0.7[0.4-1.1]$ & $0.7[0.5-1.2]$ & 0.226 & 0.694 \\
\hline Thrombocytes [G/l] & 174 [129-279] & 179 [135-292] & $177[127-236]$ & 0.415 & 0.006 \\
\hline \multicolumn{6}{|l|}{ Specific medication } \\
\hline Glucocorticoids $^{\S}$ & $120(100 \%)$ & $48(100 \%)$ & $72(100 \%)$ & 1.000 & N/A \\
\hline Remdesivir & $19(17 \%)$ & $8(17 \%)$ & $11(15 \%)$ & 0.796 & 0.215 \\
\hline Hydroxychloroquin & $15(13 \%)$ & $6(13 \%)$ & $9(13 \%)$ & 1.000 & \\
\hline Tocilizumab & $6(5 \%)$ & $4(8 \%)$ & $2(3 \%)$ & 0.403 & N/A \\
\hline
\end{tabular}

Data are reported as medians [25th-75th percentile] or as absolute counts (\%)

$p$ denotes $p$ values before ITPW weighting, $p_{\text {IPTW }}$ denotes $p$ values after IPTW adjustment

$p$-values are either from rank-sum tests, $X^{2}$-tests, or Fisher's exact tests, as appropriate

CVP: convalescent plasma; BMI: body mass index; ICU: intensive care unit; mSOFA: modified sequential organ failure assessment; PEEP: positive end expiratory pressure; vVECMO: veno-venous extracorporal membrane oxygenation; IV: invasive ventilation; NIV: non-invasive ventilation; HFNC: high flow nasal cannula; IL-6: interleukin 6; CRP: C-reactive protein; hs-TnT: high sensitive troponin T, SARS-CoV2: severe acute respiratory syndrome corona virus 2

${ }^{\$}$ Documented coronary heart disease either by specific coronary imaging or coronary angiography

${ }^{\dagger}$ Comprises immunosuppressive medication (low dose of glucocorticoids are excluded) as well as diseases with severe immunosuppression

${ }^{\S}$ Glucocorticoids included low-dose dexamethasone or equivalent doses of other glucocorticoids

${ }^{*}$ Reports variables with missing values: IL-6 - 118 patients fully observed ( $2 \%$ missing), Ferritin -118 patients fully observed ( $2 \%$ missing), hs-TnT- 119 patients fully observed ( $1 \%$ missing), D-Dimer-119 patients fully observed ( $1 \%$ missing), SARS-CoV-2-antibody positivity at ICU admission-100 patients fully observed ( $17 \%$ missing)

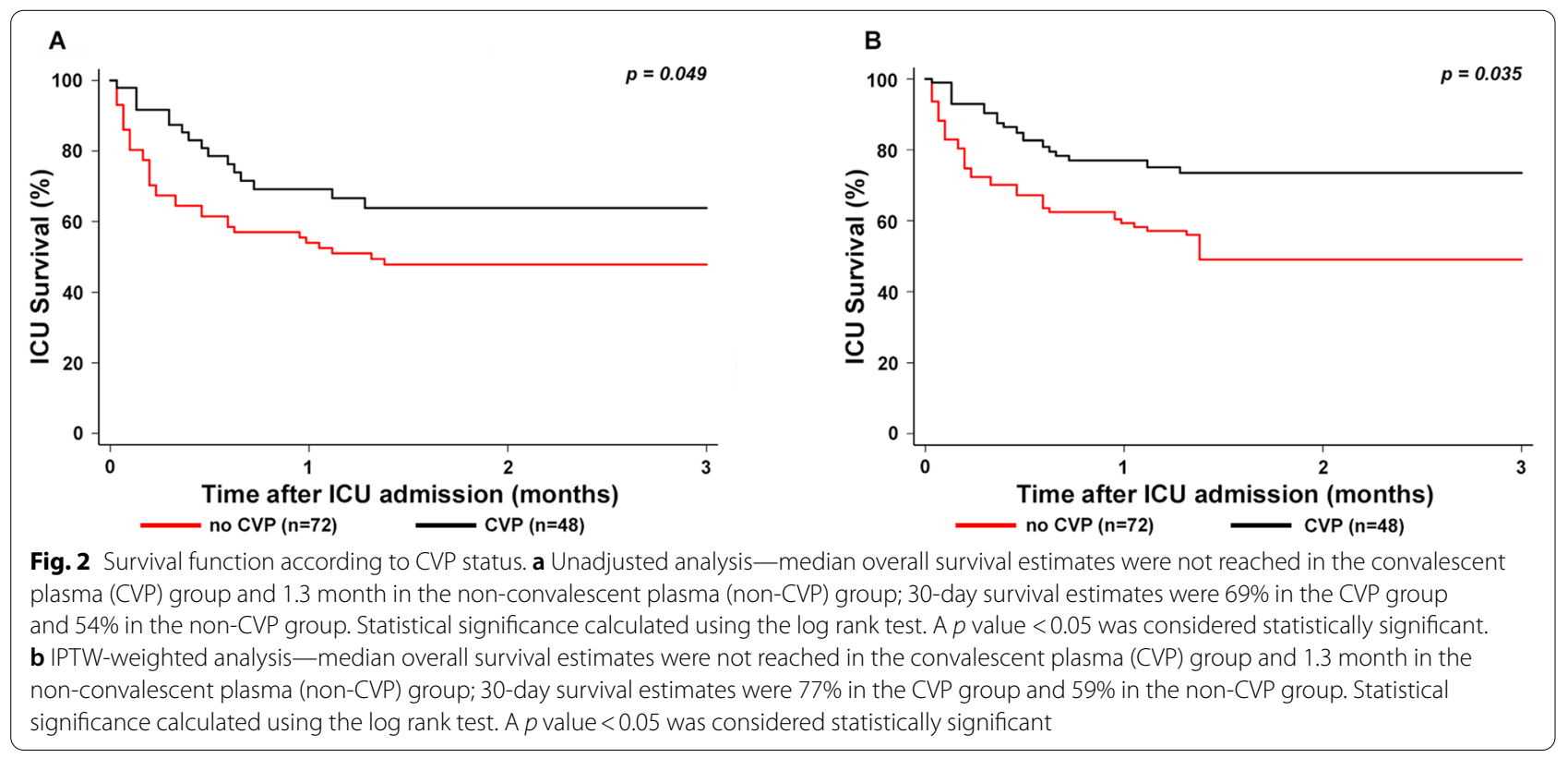

we considered to be indicative of adequate balance that requires additional multivariable adjustment for selected variables (immunosuppression, SARS-CoV-2 antibody positivity, $\mathrm{paO}_{2} / \mathrm{FiO}_{2}$ ratio, and "first wave" of COVID-19) within a sensitivity analysis.

\section{Overall survival according to CVP administration-IPTW analysis}

We then sought to reduce non-random assignment effects within our study population. Therefore, we weighted the time-to-event data for the IPTW and could demonstrate that the favorable association between 
Table 2 Propensity score model for treatment group assignment

\begin{tabular}{|c|c|c|c|}
\hline Variable & Multivariable odds ratio (OR) & $95 \% \mathrm{Cl}$ & $p$ \\
\hline \multicolumn{4}{|l|}{ Demographic variables } \\
\hline Age (per 5 years increase) & 0.67 & $0.51-0.89$ & 0.006 \\
\hline "Firstwave" of COVID-19 (per 30-day increase) & 1.80 & $1.31-2.48$ & $<0.0001$ \\
\hline Number of comorbidities (per 1 condition increase) & 1.98 & $1.27-3.07$ & 0.002 \\
\hline Chronic renal failure & 0.16 & $0.02-1.04$ & 0.05 \\
\hline Active malignancy & 8.03 & $0.26-250.22$ & 0.235 \\
\hline $\mathrm{paO}_{2} / \mathrm{FiO}_{2}$ (per 10 units increase) & 0.72 & $0.60-0.87$ & 0.001 \\
\hline Lactate (per 1 mmol/l increase) & 0.77 & $0.60-0.99$ & 0.038 \\
\hline CRP (per $50 \mathrm{mg} / \mathrm{dl}$ increase) & 1.44 & $0.98-2.12$ & 0.063 \\
\hline Ferritin (per 1000 ng/ml increase) & 1.25 & $0.95-1.64$ & 0.107 \\
\hline Absolute neutrophil count (per $1 \mathrm{G} /$ increase) & 0.76 & $0.63-0.92$ & 0.004 \\
\hline
\end{tabular}

CVP and OS became even stronger (IPTW-weighted log-rank $p=0.035$, Fig. 2b). We calculated the 30-day OS after weighting the time-to event data which were $77 \%$ in the CVP group, and 59\% in the non-CVP group. This corresponded to an estimated 2.3-fold lower risk of death (IPTW-adjusted HR $=0.44,0.21-0.95, p=0.035$ ) after CVP therapy. This association prevailed after multivariable adjustment for covariates with clear prognostic relevance (Additional file 4) and/or an insufficiently reduced imbalance between the two treatment groups (as reported in Table 1), and/or variables that by local guidance led to a recommendation for CVP administration (immunosuppression, negativity for SARS-CoV-2 antibodies, Table 3). Here, besides CVP, the strongest multivariable independent predictor for a more favorable
OS was positivity for SARS-CoV-2 antibodies at ICU admission. Even though stringent inclusion criteria were applied for CVP allocation three patients did not receive CVP despite a negative anti-SARS-CoV-2 antibody status (Table 1) allowing this analysis.

Impact of CVP antibody concentration and time to infusion In those 48 patients receiving CVP therapy, higher antibody concentrations did not emerge as a predictor of more favorable survival outcome (HR per $50 \mathrm{AU} / \mathrm{ml}$ increase $=1.07,95 \%$ CI $0.62-1.85, p=0.815)$. Similarly, time from diagnosis of COVID-19 to CVP infusion was not associated with survival at 3 months (HR per 5-day increase $=0.70,95 \%$ CI $0.41-1.19, p=0.187$ ).

Table 3 Multivariable Cox regression of overall survival according to convalescent plasma therapy in the overall cohort $(n=118)$

\begin{tabular}{|c|c|c|c|c|}
\hline Multivariable model & Variable & $\begin{array}{l}\text { Multivariable hazard } \\
\text { ratio }\end{array}$ & $95 \% \mathrm{Cl}$ & $p$ \\
\hline \multirow[t]{2}{*}{$\# 1(n=118)$} & CVP & 0.39 & $0.19-0.81$ & 0.011 \\
\hline & $\mathrm{paO}_{2} / \mathrm{FiO}_{2}$ (per 10 units increase) & 0.87 & $0.80-0.95$ & 0.002 \\
\hline \multirow[t]{2}{*}{$\# 2(n=118)$} & CVP & 0.39 & $0.18-0.84$ & 0.016 \\
\hline & mSOFA (per 1 point increase) & 1.26 & $1.08-1.46$ & 0.003 \\
\hline \multirow[t]{2}{*}{$\# 3(n=118)$} & CVP & 0.29 & $0.13-0.66$ & 0.003 \\
\hline & Positivity for SARS-COV-2 antibodies & 0.46 & $0.22-0.95$ & 0.036 \\
\hline \multirow[t]{2}{*}{$\# 4(n=118)$} & CVP & 0.38 & $0.17-0.85$ & 0.018 \\
\hline & Immunosuppression & 1.75 & $0.65-4.68$ & 0.265 \\
\hline \multirow[t]{4}{*}{$\# 6(n=98)$} & CVP & 0.21 & $0.09-0.47$ & $<0.0001$ \\
\hline & Positivity for anti-SARS-COV-2 antibodies & 0.39 & $0.18-0.85$ & 0.018 \\
\hline & mSOFA (per 1 point increase) & 1.30 & $1.10-1.54$ & 0.003 \\
\hline & Immunosuppression & 1.40 & $0.51-3.81$ & 0.515 \\
\hline
\end{tabular}

Two patients were excluded because of missing variables. Model\#1 is CVP adjusted for $\mathrm{paO}_{2} / \mathrm{FiO}_{2}$, Model\#2 is CVP adjusted for mSOFA-modified sequential organ failure assessment, Model\#3 is CVP adjusted for anti-SARS-CoV-2 antibodies positivity at time of ICU admission, Model\#4 is CVP adjusted for immunosuppression at time of ICU admission, Model\#5 is CVP adjusted for anti-SARS-CoV-2 antibodies positivity at time of ICU admission, mSOFA and immunosuppression In these analyses, the association between CVP and a higher risk of death prevailed after multivariable adjustment 


\section{Exploratory analysis of predictive markers}

In subgroup analyses using IPTW-adjusted Cox models, the association between CVP and favorable survival outcome was consistent across pre-specified subgroups defined by age (interaction $p=0.496)$, comorbidities $(p=0.800)$, mSOFA score $(p=0.600)$, and ferritin levels $(p=0.570)$, but not across the $\mathrm{paO}_{2} / \mathrm{FiO}_{2}$ ratio (Fig. 3). Here, CVP appeared to interact with the $\mathrm{paO}_{2} /$ $\mathrm{FiO}_{2}$ ratio (interaction $p=0.033$ ), which prompted us to explore the potential modifying "effect" of invasive ventilation on the association between CVP and survival. In this hypothesis generating, post-hoc analysis, the potential positive effect of CVP appeared to be confined to patients on non-invasive ventilation (Fig. 3). In detail, IPTW-adjusted HRs for CVP were 0.12 (95\% CI $0.03-0.57, p=0.007)$ in those with non-invasive ventilatory support, and $1.10(0.54-2.22, p=0.798)$ in those with invasive ventilatory support, respectively (interaction $p=0.011$ ).

\section{Discussion}

Within this prospective cohort study CVP therapy was associated with improved OS in severe to life-threatening diseased COVID-19 patients with absence of antiSARS-CoV-2 antibody seroconversion at time of ICU admission. The survival benefit of CVP therapy was even evident in crude analyses although patients receiving CVP had an increased risk profile compared to those not selected for this treatment. In addition, we were able to highlight that absence of anti-SARS-CoV-2 antibodies in patients at time of ICU admission is one of the strongest multivariable predictors of worse ICU outcomes in our cohort, strengthening the rational of our in-house defined protocol to treat those patients with CVP.

The presence of anti-SARS-CoV-2 antibodies indicates an active targeted immune response with the potential to limit viral spread. Patients unable to mount a timely antibody response may be in disadvantage as they rely merely on the anti-viral capacities of innate immune cells
Variable and Subgroup

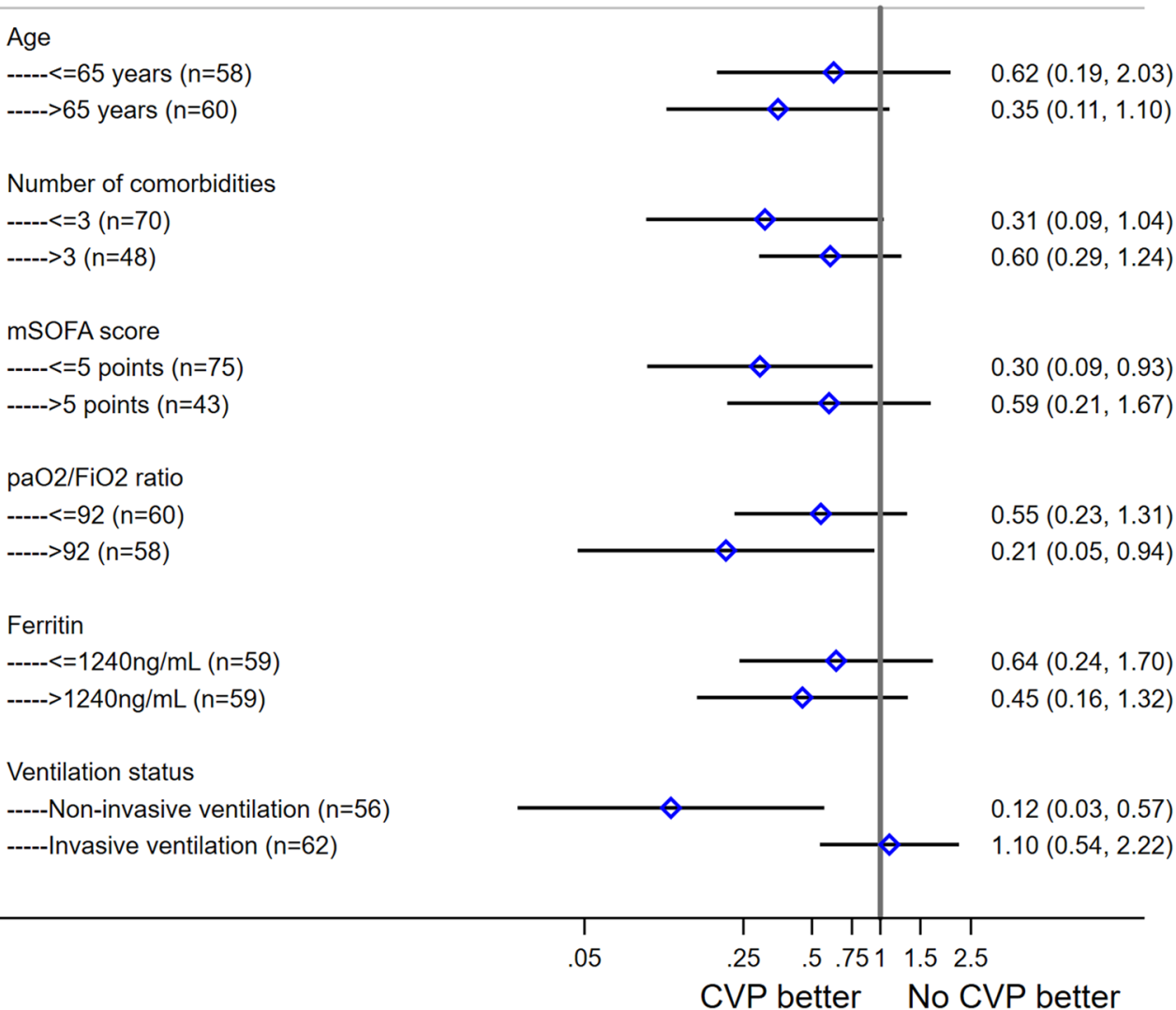

Fig. 3 Subgroup analysis - forest plot of the relative association of CVP with overall survival according to selected clinical co-variables. Hollow-blue diamonds represent the subgroup hazard ratio, and the associated bars the $95 \%$ confidence interval. The black vertical line represents the "line of unity", at which patients who were and were not treated with CVP have similar hazards of overall mortality. Regression results were obtained by fitting an interaction between the CVP treatment and the respective subgroup variable 
and T cells [24-26]. Our herein provided beneficial outcome results for patients receiving CVP are consistent with aggregated data from non-randomized and randomized clinical trials showing beneficial outcomes with regard to CVP [13, 15, 17, 18]. Similarly, those studies focused on special characterized cohorts to tailor a personalized treatment for critically ill COVID-19 patients. Our patients received CVP early after onset of symptoms (median 4 days; 25 th-75th percentile: $1-10$ ). This fact agrees with clinical trials showing favourable outcomes due to CVP $[13,15,17,18]$ but is in contrast to those who failed underlining the need for more data on this topic as provided by our investigation $[12,14,16,27$, 28]. In addition, two large clinical trials could highlight the importance of high anti-SARS-CoV-2 antibody concentrations of CVP as well as earlier administration of CVP in the course of the disease $[17,18]$. Interestingly, neither higher antibody concentration of CVP nor time from COVID-19 diagnosis to CVP infusion were associated with better CVP effects. This fact might be biased by case allocation driven by sero-positivity of patients which vanishes the effect of higher antibody concentration as well as early severity of COVID-19 which vanishes the effect of time to infusion.

We could demonstrate that ICU treatment during the first wave of COVID-19 was an independent negative prognostic marker of ICU outcomes (Table 2). Similar findings of declining ICU mortality during the pandemic waves have been published by other groups and might reflect learning processes and deeper knowledge of disease mechanisms as well as an optimization of ventilation concepts and other ICU treatment strategies [29, 30]. However, any potential time dependent bias of CVP administration has been eliminated by our statistical calculations using IPTW weighted analysis.

In an exploratory analysis we could demonstrate that patients with non-invasive ventilation benefit most from CVP therapy. One might speculate that more severe acute respiratory failure requiring invasive ventilation has advanced from an inflammatory to a more proliferative phase. While proliferative alveolar damage is frequently found upon autopsy of COVID-19 cases, anti-viral therapy such as CVP might not be able to improve the course of disease at that stage [31]. Therefore, our findings suggest that those patients receiving non-invasive ventilation should be rapidly evaluated for CVP treatment after ICU admission to reduce the risk of intubation and improve their OS.

Limitations of our study are its observational nature, the imbalances of the patient characteristic regarding their specific risk profiles and the relatively long timeframe of study inclusion, which might demonstrate a bias as discussed above. However, we can provide high data quality by prospective collection and inclusion in our registry and we used very stringent inclusion criteria to reduce any bias. To account this non-random and time depended assignment effects of our study population we performed a propensity score adjusted analysis which has been described as a potent statistical method in the field of critical care [32]. By this analysis we were able to balance the risk factors between both groups and generate a sufficient pseudo-population which further strengthened our finding of beneficial effects of CVP on ICU mortality.

To the best of our knowledge, our investigation is the first prospective cohort study evaluating CVP therapy on OS in a severely ill and well characterized ICU cohort. Importantly, the baseline as well as ICU specific characteristics of our study population were demonstrate the sickest subgroup of a large recently published European ICU cohort comprising more than 4000 patients [30]. This supports the relevance and generalizability of our findings to all disciplines in charge of COVID-19 patient care.

\section{Conclusion}

In this observational study comprising 120 critically ill patients and high proportion of immunocompromised patients with PCR confirmed COVID-19 and associated acute respiratory failure admitted to our ICUs, we were able to demonstrate that CVP treatment is able to improve ICU outcomes especially in patients with absence of anti-SARS-CoV-2 antibodies at time of ICU admission. We could further strengthen this finding using propensity scores to balance the population for known and unknown risk factors. In summary, we report the utility of CVP in a "real-world" ICU-cohort of critically ill COVID-19 patients highlighting the potential relevance of our finding to the field of intensive care medicine.

\section{Supplementary Information}

The online version contains supplementary material available at https://doi. org/10.1186/s13613-021-00867-9.

Additional file 1: Table S1. Tabulation of immunocompromised patients. R-CHOP21 - combination chemotherapy consisting of rituximab, cyclophosphamide, vincristine, prednisolone given in 21-day cycles; FOLFOX combination chemotherapy consisting of folic acid, 5-fluorouracil and oxaliplatin; DA 7 +3-combination chemotherapy consisting of cytarabine given continuously for 7 days and daunorubicin given on 3 consecutive days.

Additional file 2. Supplementary Materials and Methods.

Additional file 3: Figure S1. Overall survival of the whole cohort ( $n=120$ ). Median follow-up of the whole cohort was 1.9 months (with follow-up truncated at 3 months), we totally observed 52 deaths which corresponds to 1-, 2-, and 3-month OS estimates of 60\% (95\% Cl 51-68), 54\% (45-63), and 54\% (45-63). Cl: confidential interval.

Additional file 4: Table S2. Univariable predictors of survival in the cohort ( $n=120)$. BMl: body mass index; first wave: depicts patients treated 
during the first wave of COVID-19 in Austria between March 2020 and August 2020; mSOFA: modified sequential organ failure assessment, maxPEEP: depicts the maximum applied PEEP to achieve optimal ventilation; IL-6: interleukin 6; CRP-C-reactive protein; hs-TNT: high sensitive troponin T

Additional file 5: Figure S2. Histograms of the Propensity Score and the IPTW. (A) The propensity score can range from 0 to 1 . Multiply by 100 to obtain probabilities (in percent) of having received CVP. (B) The IPTW was defined as the inverse of the probability of receiving the treatment that the patient received (i.e., the so-called "average treatment effect on the treated

Additional file 6: Figure S3. Standardized mean difference (SMD) plot. Blue squares denote Standardized mean differences (SMDs) in before weighting the inverse of the probability of treatment weight (IPTW). Yellow diamonds denote the SMDs after weighting the IPTW.

\section{Acknowledgements}

\section{Not applicable.}

\section{Authors' contributions}

SH, RK, PS and GH designed the study. SH, FP developed the methodology and performed statistical analysis. SH, RK, GS, ZS, FP, NS, KR and PS collected data. SH, FP, RK and GS analyzed and interpreted the data. SH, RK and GS drafted the paper. MS, ACR, PE, MS, WT, ZS, FP, KG, SP, ARR, HTG and PS revised the paper. SH and FP provided technical and administrative support. SH, RK and GS supervised the study. All authors read and approved the final manuscript.

\section{Funding}

City of Graz Research Grant to SH.

\section{Availability of data and materials}

Full patient data set and STATA analysis code are available on request by SH.

\section{Declarations}

\section{Ethical approval and consent to participate}

All procedures performed in studies involving human participants were in accordance with the ethical standards of the institutional and/or national research committee and with the 1964 Helsinki declaration and its later amendments or comparable ethical standards. Approval of the local institutional review board: (EK-Nr.: 32-475 ex 19/20).

\section{Consent for publication}

Not applicable.

\section{Competing interests}

None of the contributing authors has any conflicts of interest, including specific financial interests and relationships and affiliations relevant to the subject matter or materials discussed in the manuscript.

\section{Author details}

${ }^{1}$ Intensive Care Unit, Department of Internal Medicine, Medical University of Graz, Graz, Austria. ${ }^{2}$ Division of Hematology, Department of Internal Medicine, Medical University of Graz, Graz, Austria. ${ }^{3}$ Division of Oncology, Department of Internal Medicine, Medical University of Graz, Graz, Austria. ${ }^{4}$ Department for Blood Group Serology and Transfusion Medicine, Medical University Graz, Graz, Austria. ${ }^{5}$ Division of Rheumatology and Immunology, Department of Internal Medicine, Medical University of Graz, Graz, Austria. ${ }^{6}$ Department of Anesthesiology and Intensive Care Medicine, Medical University Graz, Graz, Austria. ${ }^{7}$ Clinical Institute for Medical and Chemical Laboratory Diagnostics, Medical University of Graz, Graz, Austria. ${ }^{8}$ Division of Angiology, Department of Internal Medicine, Medical University of Graz, Graz, Austria. ${ }^{9}$ Division of Endocrinology and Diabetology, Department of Internal Medicine, Medical University of Graz, Graz, Austria. ${ }^{10}$ Division of Nephrology, Department of Internal Medicine, Medical University of Graz, Graz, Austria. ${ }^{11}$ Section of Infectious Diseases and Tropical Medicine, Department of Internal Medicine, Medical University of Graz, Graz, Austria.
Received: 14 March 2021 Accepted: 4 May 2021

Published online: 12 May 2021

\section{References}

1. Bauer J, Brüggmann D, Klingelhöfer D, et al. Access to intensive care in 14 European countries: a spatial analysis of intensive care need and capacity in the light of COVID-19. Intensive Care Med. 2020;46:2026-34.

2. Definition Task Force ARDS, Ranieri VM, Rubenfeld GD, et al. Acute respiratory distress syndrome: the Berlin Definition. JAMA. 2012;307:2526-33.

3. Beigel JH, Tomashek KM, Dodd LE, et al. Remdesivir for the Treatment of Covid-19 - Final Report. N Engl J Med. 2020;383:1813-26.

4. RECOVERY Collaborative Group, Horby P, Lim WS, et al. Dexamethasone in Hospitalized Patients with Covid-19. N Engl J Med. 2021;384(8):693-704. https://doi.org/10.1056/NEJMoa2021436.

5. Cao B, Wang Y, Wen D, et al. A trial of lopinavir-ritonavir in adults hospitalized with severe Covid-19. N Engl J Med. 2020;382:1787-99.

6. RECOVERY Collaborative Group. Effect of hydroxychloroquine in hospitalized patients with Covid-19. N Engl J Med. 2020;383:2030-40.

7. Salama C, Han J, Yau L, et al. Tocilizumab in patients hospitalized with Covid-19 Pneumonia. N Engl J Med. 2021;384:20-30.

8. Yiğenoğlu TN, Hacıbekiroğlu T, Berber l, et al. Convalescent plasma therapy in patients with COVID-19. J Clin Apher. 2020;35:367-73.

9. Sanders JM, Monogue ML, Jodlowski TZ, et al. Pharmacologic treatments for coronavirus disease 2019 (COVID-19). A review. JAMA. 2020;323:1824-36.

10. Mangge $H$, Prüller F, Schnedl W, et al. Beyond macrophages and T cells: $B$ cells and immunoglobulins determine the fate of the atherosclerotic plaque. Int J Mol Sci. 2020;21:4082. https://doi.org/10.3390/ijms21114082.

11. Joyner MJ, Bruno KA, Klassen SA, et al. Safety update: COVID-19 convalescent plasma in 20,000 hospitalized patients. Mayo Clin Proc. 2020;95:1888-97.

12. Simonovich VA, Burgos Pratx LD, Scibona P, et al. A randomized trial of convalescent plasma in Covid-19 severe Pneumonia. N Engl J Med. 2020;384(7):619-29.

13. Liu STH, Lin H, Baine I, et al. Convalescent plasma treatment of severe COVID-19: a propensity score-matched control study. Nat Med. 2020;26:1708.

14. Agarwal A, Mukherjee A, Kumar G, et al. Convalescent plasma in the management of moderate covid-19 in adults in India: open label phase II multicentre randomised controlled trial (PLACID Trial). BMJ. 2020;371:m3939.

15. Salazar E, Christensen PA, Graviss EA, et al. Treatment of coronavirus disease 2019 patients with convalescent plasma reveals a signal of significantly decreased mortality. Am J Pathol. 2020;190:2290-303.

16. Li L, Zhang W, Hu Y, et al. Effect of convalescent plasma therapy on time to clinical improvement in patients with severe and life-threatening COVID-19. A randomized clinical trial. JAMA. 2020;324:460-70.

17. Libster R, Pérez Marc G, Wappner D, et al. Early high-titer plasma therapy to prevent severe Covid-19 in older adults. N Engl J Med. 2021;384(7):610-8.

18. Joyner MJ, Carter RE, Senefeld JW, et al. Convalescent plasma antibody levels and the risk of death from Covid-19. N Engl J Med. 2021;384(11):1015-27.

19. Hatzl S, Eisner F, Schilcher G, et al. Response to "COVID-19 in persons with haematological cancers." Leukemia. 2020;34:2265-70.

20. National SARS-CoV-2 Serology Assay Evaluation Group. Performance characteristics of five immunoassays for SARS-CoV-2: a head-to-head benchmark comparison. Lancet Infect Dis. 2020;20:1390-400.

21. Austin PC. An introduction to propensity score methods for reducing the effects of confounding in observational studies. Multivariate Behav Res. 2011;46:399-424

22. Austin PC, Stuart EA. Moving towards best practice when using inverse probability of treatment weighting (IPTW) using the propensity score to estimate causal treatment effects in observational studies. Stat Med. 2015;34:3661-79.

23. White IR, Daniel R, Royston P. Avoiding bias due to perfect prediction in multiple imputation of incomplete categorical variables. Comput Stat Data Anal. 2010;54:2267-75.

24. Wölfel R, Corman VM, Guggemos W, et al. Virological assessment of hospitalized patients with COVID-2019. Nature. 2020;581:465-9. 
25. Wellinghausen N, Plonné D, Voss M, et al. SARS-CoV-2-IgG response is different in COVID-19 outpatients and asymptomatic contact persons. J Clin Virol. 2020;130:104542.

26. Weinreich DM, Sivapalasingam S, Norton T, et al. REGN-COV2, a neutralizing antibody cocktail, in outpatients with Covid-19. N Engl J Med. 2021;384:238-51.

27. Gharbharan A, Jordans CCE, Geurtsvankessel C, et al. 2020. Convalescent Plasma for COVID-19. A randomized clinical trial. medRxiv. 2020.07.01.20139857.

28. Avendano-Sola C, Ramos-Martinez A, Munez-Rubio E, et al. 2020. Convalescent Plasma for COVID-19: A multicenter, randomized clinical trial. medRxiv. 2020.08.26.20182444.

29. Auld SC, Caridi-Scheible M, Robichaux C, et al. Declines in mortality ove time for critically ill adults with coronavirus disease 2019. Crit Care Med. 2020;48:e1382-4.
30. COVID-ICU Group on behalf of the REVA Network and the COVID-ICU Investigators. Clinical characteristics and day-90 outcomes of 4244 critically ill adults with COVID-19: a prospective cohort study. Intensive Care Med. 2021;47:60-73.

31. Carsana L, Sonzogni A, Nasr A, et al. Pulmonary post-mortem findings in a series of COVID-19 cases from northern Italy: a two-centre descriptive study. Lancet Infect Dis. 2020;20:1135-40.

32. Gayat E, Pirracchio R, Resche-Rigon M, et al. Propensity scores in intensive care and anaesthesiology literature: a systematic review. Intensive Care Med. 2010;36:1993-2003.

\section{Publisher's Note}

Springer Nature remains neutral with regard to jurisdictional claims in published maps and institutional affiliations.

\section{Submit your manuscript to a SpringerOpen ${ }^{\circ}$ journal and benefit from:}

- Convenient online submission

- Rigorous peer review

- Open access: articles freely available online

- High visibility within the field

- Retaining the copyright to your article

Submit your next manuscript at springeropen.com 